\title{
Littératies multimodales et perspectives critiques
}

\section{Diane Dagenais}

\section{(2) OpenEdition}

\section{Journals}

Édition électronique

URL : http://journals.openedition.org/rdlc/2338

DOI : $10.4000 /$ rdlc. 2338

ISSN : $1958-5772$

Éditeur

ACEDLE

\section{Référence électronique}

Diane Dagenais, «Littératies multimodales et perspectives critiques », Recherches en didactique des langues et des cultures [En ligne], 9-2 | 2012, mis en ligne le 03 octobre 2017, consulté le 10 décembre 2020. URL : http://journals.openedition.org/rdlc/2338; DOI : https://doi.org/10.4000/rdlc.2338

Ce document a été généré automatiquement le 10 décembre 2020.

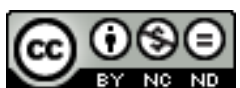

Recherches en didactique des langues et des cultures is licensed under a Creative Commons AttributionNonCommercial-NoDerivatives 4.0 International License 


\title{
Littératies multimodales et perspectives critiques
}

\author{
Diane Dagenais
}

Nous sommes reconnaissante au Dr. Mario López Gopar d'avoir facilité l'accès au site mexicain et pour sa précieuse collaboration tout au long de ce projet. Nous voulons également reconnaître la contribution inestimable des stagiaires mexicains et canadiens qui ont investi beaucoup d'énergie dans la mise en œuvre de ce projet et la collaboration sur le terrain en fournissant aussi leurs interprétations intuitives et informées des activités de littératies multimodales. Finalement, nous remercions les enfants et leurs parents qui ont accepté de participer à ce projet et de partager leurs connaissances.

1 Avec la multiplication des télécommunications numériques ces dernières années, les recherches sur les pratiques de littératie dépassent maintenant l'étude des interactions autour de textes écrits pour prendre en compte la grande diversité des formes de communication intégrant plusieurs moyens d'expression : l'oral, l'écrit, la musique, la gestuelle, le son, l'image fixe et mobile, et la gestion de l'espace. Dans les sociétés du 21e siècle où les outils numériques circulent largement, ces types de communication prennent une plus grande importance dans les échanges sur les places publiques et dans les espaces privés, comme la maison, ce qui permet aux enfants de participer régulièrement à ces pratiques hybrides de littératie et d'acquérir des compétences dans ce domaine dès un jeune âge (Dagenais \& Moore, 2008; Gregory \& Williams, 2000). Certains ont aussi l'opportunité de développer ces compétences davantage à l'école quand les approches pédagogiques en place encouragent les enfants à explorer, analyser et utiliser de façon pertinente et informée une plus grande variété de modes d'expression qui font partie de la vie quotidienne dans plusieurs contextes.

2 Afin de clarifier comment l'étude des littératies prend en compte aujourd'hui une plus grande diversité expressive, nous présentons une discussion des développements théoriques dans le domaine en nous concentrant sur plusieurs termes, dont les littératies multiples, littératies plurilingues, répertoire plurilittératié, multimodalité et littératies multimodales, qui circulent depuis quelques temps dans les écrits scientifiques en anglais et qui sont apparus récemment dans des publications en français. Par la suite, nous 
décrivons comment une perspective critique a orienté les travaux de chercheurs qui considèrent que les pratiques de littératies multimodales en milieu d'apprentissage sont situées dans le cadre plus large des dynamiques de pouvoir dans la société. Enfin, nous examinons quelques extraits de données provenant d'un projet de production et d'échange de vidéos pour montrer comment une perspective critique peut servir à éclairer les possibilités et les limites d'une innovation pédagogique axée sur les littératies multimodales.

\section{Développements théoriques}

\section{Perspectives sociales sur les pratiques de littératie}

3 Le terme littératie (Painchaud, d'Anglejan, Armand, \& Jesak, 1993) a été introduit en français, il y a environ vingt ans, par une équipe de recherche québécoise œuvrant en didactique des langues secondes qui cherchait à élargir le champ conceptuel adopté jusque-là dans la communauté scientifique francophone pour étudier l'apprentissage de la lecture et de l'écriture. En proposant ce néologisme, ces chercheures voulaient tenir compte de la façon dont le terme équivalent en anglais (literacy) avait évolué dans les décennies précédentes pour désigner l'usage de l'écrit dans son sens large. En effet, les définitions de la littératie qui circulaient depuis plusieurs années dans les milieux anglophones se référaient à la fois aux aspects individuels et cognitifs de la compréhension et de la production de textes écrits ainsi qu'aux dimensions sociales des interactions adoptées autour de l'écriture et aux fonctions attribuées à l'écrit dans différentes sociétés (Ferdman, 1990). Cette conception de la littératie s'est développée à la lumière des résultats de recherches ethnographiques inspirées de traditions anthropologiques et sociolinguistiques montrant que la littératie en contexte naturel et scolaire est fondamentalement un processus social de communication axé sur la construction de sens (Wells, 1986). Écrit en français avec des terminaisons différentes, tie ou -cie, selon les auteurs (Chiss, 2003; Armand, 2000 ; Dagenais \& Moore, 2008 ; Fraenkel \& Mbodj, 2010 ; Moore, 2006 ; Reuter, 2003), le terme littératie est maintenant assez répandu et utilisé pour se référer à un large éventail de pratiques contextualisées, fonctionnelles et sociosémiotiques.

4 C'est dans cette optique que s'insèrent les écrits de la lignée des Nouvelles études de la littératie (New Literacy Studies) où celle-ci est définie comme un ensemble de processus sociaux, culturels et académiques mis en place au sein d'une communauté discursive (Street, 2000 et 2003). Les chercheurs associés à ce courant visent à décrire les pratiques de littératie (literacy practices) à partir d'une perspective locale et de l'interprétation émique que les participants impliqués dans des activités de communication accordent à celles-ci. Leurs études, menées dans une grande variété de milieux sociaux, fournissent des données comparatives sur les pratiques situées. En se basant sur l'observation d'activités de littératie quotidiennes menées à l'intérieur et à l'extérieur de l'école, plusieurs chercheurs se sont attardés plus spécifiquement à identifier quelles pratiques sont valorisées dans des contextes particuliers et lesquelles sont marginalisées pour éclairer comment les forces à l'œuvre dans la société plus large ont un impact sur les pratiques locales.

5 Toutefois, une centration trop étroite sur le local dans ces études a été critiquée par Brandt et Clinton (2000) qui indiquent qu'elle risque d'éclipser comment les objets 
matériels produits par les pratiques de littératie circulent dans le monde et perdurent avec le temps (pour une discussion plus poussée, voir Van Enk, Dagenais \& Toohey, 2005). Les possibilités et les limites des Nouvelles études de la littératie ont fait l'objet dernièrement d'un numéro de Langage et société, où Fraenkel et Mbodj (2010) présentent une discussion nuancée de l'intérêt que présente ce champ d'étude pour la francophonie ainsi qu'une traduction en français de quelques publications importantes dans ce domaine.

Selon Street (2000 et 2003), les New Literacy Studies reposent sur un modèle idéologique qui s'oppose à une vision autonome, laquelle conçoit la littératie comme une habileté neutre, un outil technique ou un facteur indépendant pouvant servir à améliorer de façon universelle les conditions de vie de tous. Elles remettent en question cette présupposition en montrant que les pratiques sont inextricablement liées aux structures de pouvoir dans les milieux où elles sont situées, qu'elles sont interprétées de façons différentes par les groupes sociaux et qu'elles varient en fonction des conditions matérielles et sociales en place. Dans ce courant, la littératie n'est pas vue de façon monolithique comme garant en soi de la mobilité sociale, de l'acquisition d'un plus grand pouvoir économique et de l'établissement de l'équité dans la société. Ces recherches considèrent plutôt comment certaines formes de littératie sont valorisées plus que d'autres dans divers lieux comme l'école, le milieu du travail et d'autres institutions sociales où elles servent d'instruments de contrôle bureaucratique et d'oppression pour maintenir les hiérarchies sociales (Carrington \& Luke, 1997). Certaines études visent donc à examiner comment les discours idéologiques sont impliqués dans la définition de la littératie comme capital nécessaire à acquérir d'autres formes de ressources sociales et matérielles. Ces études permettent de dépister où se situent les tensions et les contradictions entre les discours sur les bénéfices de la littératie et les pratiques adoptées localement dans le quotidien.

\section{Littératies multiples, littératies plurilingues et répertoire plurilittératié}

7 Durant l'émergence des Nouvelles études de la littératie, un groupe de dix chercheurs provenant de plusieurs disciplines se nommant le New London Group $(1996$; 2000) s'est réuni aux Etats-Unis pour explorer comment reconceptualiser la littératie étant donné les mutations importantes dans les moyens de communication et les changements dans les populations des sociétés occidentales. Dans une publication collective, ils ont pris position contre la vision autonome de la littératie centrée sur la communication graphique en proposant comme alternative la notion de littératies multiples ( multiliteracies), écrite au pluriel pour souligner que la communication est caractérisée par plusieurs formes de représentation. Comme Cope et Kalantzis (2000) l'ont rappelé, cette pluralité dans la communication n'est pas nouvelle car avant le développement de l'écrit, les êtres humains utilisaient le dessin, la peinture et les icônes pour transmettre des informations sur leur vécu quotidien, leurs pratiques sociales, et leurs rites religieux. Ils rappellent aussi que les premiers manuscrits étaient généralement illustrés par des dessins qui servaient à ajouter d'autres informations au message véhiculé par l'écrit et que ce n'est qu'avec l'introduction des presses de l'imprimerie au $15^{\mathrm{e}}$ siècle que le texte écrit a pris une plus grande importance, puis que l'école s'est ensuite concentrée exclusivement sur le développement de l'écriture en laissant de côté les autres formes de représentation, ou du moins en les considérant tout simplement comme des outils 
d'expression esthétique et ludique. Comme le signale López Gopar (2007), cette compréhension plus large des pratiques de communication repose aussi sur une conception plus souple de ce que constitue le texte en considérant que celui-ci peut être imprimé, qu'il peut circuler de façon électronique dans des échanges synchrones ou asynchrones, qu'il peut être présenté en face à face (live) et paraître dans une, deux, ou plusieurs langues de façon simultanée ou alternée.

C'est pour cette dernière raison que Martin-Jones et Jones (2000) parlent de littératies plurilingues (multilingual literacies) pour signaler que les individus et les groupes ont recours à plusieurs langues pour s'exprimer. En se situant à l'intersection des New Literacy Studies et des recherches sociolinguistiques sur le bilinguisme, Martin-Jones et Jones proposent ce terme pour mieux représenter comment les pratiques communicatives émergent dans des situations de plurilinguisme où « different languages, language varieties and scripts add other dimensions to the diversity and complexity of literacies " (Martin-Jones \& Jones, 2000: 5). Dans une publication antérieure (Dagenais \& Moore, 2008), nous nous sommes inspirées de ces arguments et nous nous sommes appuyées sur le modèle de répertoire plurilingue (Coste, Moore \& Zarate, 1997; Grosjean, 1993) ainsi que sur les notions de compétences pluriscripturales et plurilittératiées (Moore, 2006), pour proposer le terme répertoire plurilittératié comme néologisme permettant d'examiner les pratiques de l'enfant plurilingue en tenant compte de "toutes ses langues, leurs diverses formes scripturales et graphiques, la diversité des modes situés de leur expression, ainsi que l'ensemble des contextes et des conditions de leur développement " (Dagenais \& Moore, 2008: 15).

\section{La multimodalité et les littératies multimodales}

Dans sa discussion sur la place prépondérante que prend le visuel, la musique ainsi que le corps et ses mouvements aujourd'hui, Kress $(2000,2003)$ avance l'argument selon lequel les théories sémiotiques focalisées sur la graphie n'expliquent pas comment ces multiples modes d'expression sont mis en place dans la communication. Il suggère que la valorisation de l'écrit comme forme privilégiée d'expression a longtemps écarté le rôle que jouent les autres sens dans la communication. Pour conceptualiser comment les pratiques sémiotiques dépassent l'interprétation des représentations graphiques du langage, il propose la notion de multimodalité (multimodality) en suggérant qu'elle permet d'élucider la contribution des divers sens dans les interactions. Pour illustrer, il explique que depuis quelques années les analyses de l'interprétation gestuelle de la langue des signes reconnaissent que les expressions du visage, les mouvements de la bouche et des yeux, la disposition des bras et des mains ainsi que l'attitude et la manipulation du haut du corps constituent un système de construction de sens coordonné à la grammaire de la langue.

D'après Kress $(2000,2003)$, il est impossible d'associer un texte à un seul mode sémiotique car plusieurs sens sont invoqués dans l'interprétation de tout type de message, y compris celui qui est écrit. Kress signale que la multimodalité des textes est encore plus évidente dans les messages représentés sur un écran électronique où les informations écrites sont transmises à partir d'une synchronisation de plusieurs modes sémiotiques tels que le son, la musique, le langage oral, et les images fixes ou mobiles. Pour analyser les interactions entre les divers modes d'expression dans la construction du message, Kress s'appuie sur une approche sémiotique qu'il avait développée au préalable avec son collègue (voir Kress 
et Van Leeuwen, 1996), basée sur la linguistique systémique fonctionnelle de Halliday (1976, 1978). Leurs analyses des textes visuels considèrent comment les ressources sémiotiques disponibles dans la société et l'importance attribuée à celles-ci dans l'imaginaire collectif orientent la construction et l'interprétation du visuel.

Plus concrètement, dans leurs analyses, Kress et Van Leeuwen (1996) examinent la manière dont les personnes et les objets qui figurent dans une représentation visuelle sont positionnés physiquement les uns par rapport aux autres dans l'espace. Ils s'attardent sur les relations entre les personnes dans le champ visuel et sur les liens établis avec ceux qui les regardent. Ils s'intéressent aussi à décrire comment les éléments de composition affectent le sens attribué au texte visuel.

Plus récemment, le terme littératie multimodale (multimodal literacy) a été présenté par Jewitt et Kress (2003) pour souligner l'aspect visuel de la littératie et pour pousser plus loin la discussion sur le rôle actif qu'adoptent les acteurs sociaux dans la construction de sens et dans l'interprétation des messages en ayant recours à plusieurs modes sémiotiques. Pour ces auteurs, comme pour ceux qui s'insèrent dans le courant des Nouvelles études de la littératie, leur recherche est centrée sur l'action des acteurs et la façon dont ces derniers utilisent les ressources sémiotiques à leur disposition dans des contextes socioculturels spécifiques. Le terme littératies multimodales (multimodal literacies) apparaît au pluriel depuis quelques années dans certaines publications (voir entre autres, Flewitt, 2008; Flood, Heath, \& Lapp ; 2008 ; Heath \& Street, 2008). C'est la formule que nous privilégions dans nos travaux car elle souligne bien la présence d'une pluralité de modes d'expression.

13 Comme le signale Hull \& Nelson (2005), pour développer les littératies multimodales, il ne s'agit pas tout simplement d'ajouter des images à des textes écrits, mais il faut reconnaître que les messages peuvent être véhiculés de plusieurs façons différentes et que les acteurs sociaux peuvent s'appuyer sur un vaste ensemble de processus sémiotiques pour les composer et les comprendre. Le recours à des littératies multimodales permet de transmettre et d'appréhender les messages échangés dans l'interaction sociale autrement que dans les situations où la communication repose seulement sur le texte écrit et illustré.

\section{Perspectives critiques appliquées à l'étude des littératies}

14 Avec l'importance que prend le social dans l'étude des littératies et l'émergence d'un modèle idéologique, les recherches dans le domaine se penchent aujourd'hui non seulement sur la description des pratiques des acteurs sociaux et des multiples ressources qu'ils empruntent dans la communication, mais elles cherchent aussi à comprendre comment les relations de pouvoir qui sous-tendent les interactions entre acteurs affectent le processus de transmission et d'interprétation des messages dans la société. Ces dynamiques intéressent aussi les chercheurs qui examinent les situations d'apprentissage, car comme l'explique Street (2003: 78) : « The ways in which teachers or facilitators and their students interact is... a social practice that affects the nature of the literacy being learned and the ideas about literacy held by the participants, especially the new learners and their position in relations of power ».

15 L'analyse critique des contextes d'apprentissage peut être rattachée aux travaux de Bourdieu et Passeron (1970) en France, Freire (1970) au Brésil, et Apple (1979) et Giroux (1983) aux Etats-Unis. Si Bourdieu et Passeron ont pu élucider quels sont les rapports 
entre l'économie, l'éducation et la langue dans la reproduction des inégalités sociales à l'école, Freire, Apple et Giroux ont pu dépasser une logique déterministe pour montrer comment certains apprenants peuvent, sous certaines conditions, se prendre en charge et contourner les inégalités du système éducatif. C'est dans cette dernière perspective que s'insèrent des recherches menées dans plusieurs domaines de l'éducation y compris la didactique des langues, regroupées sous le label "pédagogies critiques" (critical pedagogies) par Norton et Toohey (2004). En visant à problématiser les situations d'apprentissage et à identifier des pistes éducatives plus équitables pour les étudiants de diverses origines, ces recherches se relient à une tradition de recherche critique relativement longue et bien établie (Pennycook, 1999 ; 2001).

Les recherches situées dans ce courant analysent les formes d'expression adoptées par les jeunes et explorent comment l'école pourrait élargir le répertoire de pratiques de littératies jugées acceptables et valables comme outils d'apprentissage. Par exemple, Stein (2004) a emprunté une perspective critique et s'est appuyé sur le concept de multimodalité pour analyser les productions orales et dramatiques d'élèves appartenant à divers groupes linguistiques inscrits au primaire en Afrique du Sud. En présumant que la construction de sens repose fondamentalement sur une forme d'interprétation corporelle, Stein a montré que les enfants utilisaient leur corps et qu'ils choisissaient plusieurs modes d'expression, dont le silence, pour exprimer de différentes façons leurs sentiments, leur imaginaire, leurs rêves et leurs traumatismes. En se basant sur ces observations, Stein a questionné le bien-fondé des approches pédagogiques limitées à l'expression écrite, surtout dans des contextes de diversité culturelle, de traditions orales, de tensions politiques et d'inégalités socioéconomiques, et elle a lancé l'appel à accorder plus de choix dans les modes d'expression disponibles à l'école pour se représenter et partager ses connaissances.

Dans la même veine, d'autres chercheurs se sont penchés sur des analyses de la distribution du pouvoir dans les pratiques de littératie à l'école pour montrer comment les apprenants provenant de groupes minoritaires intègrent des modes d'expression tirés du cinéma et du hip-hop dans leurs pratiques de littératie afin de résister à la marginalisation et d'établir une position plus forte dans leurs interactions avec leurs pairs. Dans une étude menée aux Etats-Unis, Hass Dyson (2001) a révélé comment des enfants issus de l'immigration cherchaient à adopter une identité désirable face aux autres en insérant des aspects de dialogues provenant de films populaires dans leurs discussions à l'école et dans les textes écrits qu'ils produisaient. Pour sa part, Ibrahim (1999) a étudié comment des immigrants francophones d'origine africaine inscrits dans une école secondaire canadienne se sont appropriés des formes discursives liées au hiphop en réaction aux identités d'étudiants noirs qui leur avaient été imposées à leur arrivée dans le pays. Ibrahim a montré comment ces adolescents se sont investis dans le hip-hop pour se positionner en tant qu'étudiants branchés et puissants. Comme Stein (2004), Hass Dyson et Ibrahim ont signalé que pour mieux soutenir l'apprentissage des jeunes de diverses origines et leur intégration dans le milieu scolaire, l'école devrait tenter de comprendre comment divers modes de communication leur offrent des options plus prometteuses pour représenter leurs connaissances et construire des identités scolaires valorisées. Notons qu'en France, sans se référer directement à la pédagogie critique ou aux littératies multimodales, des chercheurs impliqués dans le développement d'activités d'éveil aux langues (Billiez, Candelier, Costa-Galligani, Lambert, Sabatier \& 
Trimaille, 2003; Billiez \& Trimaille, 2001) avaient de leur côté proposé une façon d'intégrer le rap à l'école.

D'ailleurs, plusieurs recherches contemporaines (Brass, 2008; Bruce, 2009; Cummins, 2006 ; Debruin-Parecki \& Klein, 2003 ; Hull \& Nelsen, 2005 ; Kendrick \& McKay, 2002 ; López Gopar, 2007 ; Lotherington, Sotoudeh, Holland \& Zentena, 2008 ; Rogers, Winters, LaMonde \& Perry, 2010) s'attardent justement sur les possibilités que présentent des approches pédagogiques incorporant les littératies multimodales. Elles présentent un corpus collectif impressionnant qui révèle comment les littératies multimodales permettent aux élèves d'exprimer individuellement et en groupe des idées qu'ils ont du mal à communiquer autrement. Dans certains cas, les élèves peuvent le faire de façon plus sophistiquée et nuancée que quand ils sont contraints à communiquer uniquement par le moyen de textes écrits. En outre, comme l'indique Bruce (2009) dans une recension de 15 études sur la production de vidéos par des adolescents, la participation aux littératies multimodales n'exclue pas les tâches écrites et elle est même associée à un gain positif dans le développement de la lecture et de l'écriture.

Dans la pratique éducative, ceux qui adoptent une plus large perspective sur les littératies et sur ce qui compte comme texte ne s'appuient pas uniquement sur la lecture de manuels scolaires et la production de copies écrites, mais ils amènent les élèves à voir comment les messages sont communiqués par plusieurs moyens, y compris les représentations dramatiques, les posters, les photos, les graphies, les images, les sites web, les CD-ROMS, les DVDs, les jeux vidéo, la production vidéo et les autres outils des technologies numérisées (Cope \& Kalantzis, 2000 ; Street, 2003). Dans ces contextes d'apprentissage où l'écrit ne constitue plus le seul mode d'expression valorisé, il ne perd toutefois pas sa place importante dans la communication, car il sert toujours d'ancrage ou de point de départ pour une grande partie des interactions entre apprenants et enseignants.

20 Comme le signalent Cope \& Kalantzis (2000) la multiplication des outils d'expression dans les situations d'apprentissage exige aussi que les apprenants développent de nouvelles compétences de littératie. Par exemple, pour comprendre les messages véhiculés sur internet, les jeunes doivent apprendre non seulement à « lire » le texte écrit, mais aussi à interpréter les représentations qui l'accompagnent, à se poser des questions critiques et éthiques sur les sources des informations communiquées (Cunningham, Many, Carver, Gunderson et Mosenthal, 2000), et à considérer l'impact qu'aura la transmission de cellesci sur divers groupes ayant des ressources et des niveaux de pouvoir différents.

\section{Littératies multimodales et rapprochement maison-école}

21 L'ethnographie de Heath (1983) menée sur dix ans pour documenter les pratiques de littératie orales, visuelles et écrites dans trois communautés (différentes et voisines), aux États-Unis est souvent citée dans les écrits sur les littératies multiples ou multimodales car elle figure parmi les premières recherches qui mettent en évidence à quel point les activités scolaires étaient réduites à des tâches de lecture et d'écriture qui ne reflétaient pas la variété des littératies pratiquées par les élèves de divers groupes culturels, ce qui servait à marginaliser ces enfants et à creuser un écart entre l'école et la maison. En se basant sur les résultats de sa recherche, Heath a proposé que l'élargissement de la vision de la littératie à l'école pourrait non seulement susciter l'intérêt des jeunes de différents milieux et mettre en valeur ce que les uns et les autres savent faire à l'extérieur de l'école, mais que cela pourrait aussi ouvrir des pistes pour contourner le décrochage scolaire. 
22 À la lumière de cette étude et des résultats d'autres recherches révélant le rôle important que jouent les parents dans la socialisation à la littératie, de nombreux programmes de littératie en milieux familial, communautaire et scolaire ont été établis pour combler l'écart entre l'école et la famille. Par contre, dans son analyse critique de ces programmes, Auerbach (1989) a montré comment certaines innovations implantées dans des milieux à forte diversité culturelle reposent sur une conception déficitaire des communautés minoritaires qu'elles servent. Ces programmes supposent que les parents, généralement issus de milieux socio-économiques défavorisés et/ou migrants, ne savent pas comment développer des pratiques de littératie chez leurs enfants (souvent bi-plurilingues) et qu'il suffit tout simplement de leur montrer comment reproduire à la maison des activités de littératie de type scolaire. Auerbach a proposé qu'à l'inverse, les programmes de littératie dans ces milieux devraient tirer profit des pratiques langagières déjà en place dans le foyer, et qu'à l'école le curriculum devrait soutenir et développer davantage ces pratiques variées. Depuis, plusieurs voix se sont ajoutées à celles de Heath (1983) et d'Auerbach (1989) pour inciter le système scolaire à élargir sa perspective sur les littératies et à incorporer les pratiques de communication acquises dans le quotidien hors scolaire (Heath \& McLaughlin, 1993 ; Hull \& Schultz, 2002 ; Hull \& Nelson, 2005 ; Marsh, 2006).

Dans cette optique, Ajayi (2009) suggère qu'une pédagogie axée sur les littératies multimodales offre une plateforme plus représentative des différentes façons de communiquer adoptées dans les sociétés pluriethniques et plurilingues. Dans sa recherche menée auprès d'apprenants d'anglais langue seconde inscrits dans une école secondaire américaine, Ajayi a examiné comment l'implantation d'une activité d'analyse d'affiches publicitaires sur les téléphones cellulaires a permis aux élèves de tisser des liens entre les pratiques de littératie qu'ils adoptaient à l'extérieur et à l'intérieur de l'école. Il montre comment cette activité a mis en relief la complexité de la littératie en montrant aux élèves qu'il existe plusieurs points d'entrée dans un texte et qu'il y a de nombreuses façons de le produire et de l'interpréter. Ajayi explique que le lecteur peut, par exemple, aborder son interprétation d'une représentation multimodale en lisant ce qui est écrit ou il peut aussi bien commencer en s'attardant sur les aspects visuels du message, sur la typographie, ou sur la mise en page.

En contexte mexicain, López Gopar (2007) défend l'idée qu'une pédagogie fondée sur la notion de multimodalité serait pertinente aussi pour l'éducation des autochtones car elle reconnaît que ces peuples ont développé des pratiques de communication sophistiquées qui font appel à d'autres ressources sémiotiques que celles qui sont privilégiées à l'école. Il explique que les systèmes d'écriture développés par les Aztèques, les Mayas, les Zapotèques et les Mixtèques combinent des images avec un système numérique, des références au calendrier lunaire, des logogrammes et des syllabes ou des mots racines. À l'origine, ces textes pouvaient être lus par des peuples qui ne parlaient pas la même langue, mais qui partageaient un cadre de référence culturel. En signalant qu'ils servaient une fonction similaire aux logos commerciaux, aux icônes d'ordinateur, et aux feux de circulation qui sont lus aujourd'hui par les membres de différents groupes linguistiques, López Gopar note que la lecture de ces codex ne dépendait pas d'une lecture linéaire de haut en bas et de gauche à droite et que l'entrée dans ces textes se faisait de différentes façons. Ces observations ont mené López Gopar à proposer que l'école s'ouvre aux littératies multimodales afin d'intégrer dans le curriculum des connaissances autochtones et des pratiques de littératie associées aux productions artistiques qui circulent dans ces communautés. Il illustre le potentiel de cette approche en donnant l'exemple d'un projet 
vidéo au Mexique où des enfants autochtones parlent des arts, tel que le tissage, pratiqués dans leur communauté. Dans les vidéos, les jeunes expliquent que les symboles reproduits dans les tissages sont porteurs de messages importants liés à des mythes culturels et à des connaissances intimes de leur environnement, basés sur une mémoire collective remontant avant la conquête de l'Amérique. Il suggère qu'une pédagogie axée sur les littératies multimodales pourrait reconnaitre non seulement que les artistes sont aussi des auteurs de textes porteurs de messages importants, mais que les peuples autochtones ont des pratiques de littératies avancées et que la maîtrise de l'alphabet n'est qu'un moyen parmi d'autres pour communiquer.

Dans le contexte canadien, Marshall et Toohey (2010) ont examiné comment les représentations visuelles de jeunes apprenants d'anglais langue seconde inscrits dans des écoles primaires à Vancouver permettaient aux enfants d'aborder des sujets controversés comme la violence politique qui ne sont pas normalement discutés à l'école. Ces chercheures ont montré comment les dessins et les vidéos d'enfants servaient comme outils pour interpréter les récits transmis par leurs parents et leurs grands-parents sur les guerres, les répressions politiques et les conflits culturels dans leurs pays d'origine. Cette démarche illustre bien comment l'inclusion des littératies multimodales dans les écoles pourrait permettre aux élèves de familles immigrantes de partager les fonds de connaissances qui se transmettent d'une génération à l'autre dans leurs familles.

\section{L'analyse critique de données visuelles}

Pour rendre plus concret comment les données visuelles provenant d'un projet pédagogique axé sur les littératies multimodales peuvent être analysées à partir d'une perspective critique, nous présentons un examen initial de quelques données recueillies lors d'une recherche sur la production vidéo menée à Vancouver en collaboration avec notre collègue Kelleen Toohey. En considérant la production vidéo comme pratique de littératie située (Prinsloo, 2005), ce projet s'appuie sur les résultats de nos recherches antérieures qui révèlent que les enfants de diverses communautés linguistiques emploient régulièrement les outils de la télécommunication numérique, comme les média de réseautage social, pour s'engager dans des échanges plurilingues où ils combinent différents types de graphie avec des textes audiovisuels en fonction de leurs besoins et des ressources à leur disposition (Dagenais, 2008; Dagenais \& Day, 1998 et 1999 ; Dagenais \& Moore, 2008 ; Smythe \& Toohey, 2009a \& b ; Marshall \& Toohey, 2010). Ce projet est lui aussi basé sur une étude pilote menée par Toohey à Vancouver qui indiquent que les activités de production vidéo stimulent le développement des compétences orales et écrites en anglais langue seconde et qu'elles encouragent les enfants à utiliser des ressources matérielles, sociales et linguistiques rarement visibles à l'école (Toohey \& Schulze, à paraître).

Les activités de production vidéo dans notre projet ont été prises en charge par des étudiants-maitres inscrits dans un programme de formation initiale à l'Université Simon Fraser pendant un stage d'enseignement dans une école primaire ou secondaire située soit à Vancouver au Canada, soit à Dharamsala en Inde, ou à Oaxaca au Mexique. Dans ces activités, qui se sont déroulées après l'école ou pendant la journée scolaire selon le site, les enfants ont représenté des aspects de leur vie dans des vidéos afin de les échanger avec les jeunes vivant dans les autres sites. L'objectif de recherche était de voir sous quelles conditions matérielles et sociales l'intégration de la production vidéo dans 
l'enseignement pourrait présenter un mode de communication bi-plurilingue utile et stimulant pour les enfants, ainsi qu'un exercice pertinent pour développer chez les étudiants-maitres des connaissances sur l'enseignement des littératies multimodales. Des notes de terrain ont été recueillies sur certaines activités de production vidéo et à leur retour sur le campus de l'université, les étudiants-maîtres ont participé à des entrevues de groupe sur le processus de mise en place de ces activités, puis certains d'entre eux ont produit des réflexions rétrospectives écrites sur le déroulement de la production vidéo et sur leur vision de l'enseignement des littératies multimodales suite à cette expérience.

$\mathrm{Au}$ moment de la rédaction de cet article, la phase d'échange des vidéos est en cours sur deux des trois sites. Une analyse plus poussée des vidéos sera effectuée dans les prochains mois à l'aide de Studiocode, un programme informatique développé spécifiquement pour coder les données vidéo (voir www.studiocodegroup.com). Nous allons poursuivre les pistes d'analyse suggérées par Kendrick, Rogers, Toohey, Marshall, Mutonyi, Hauge, Siegel \& Rowsell (2010) pour examiner quels types de matériel visuel et textuel sont présents dans les vidéos, quels messages celles-ci véhiculent et quelles présuppositions sont évidentes concernant ceux qui vont les visionner. Nous avons l'intention de nous référer aux outils heuristiques proposés par Van Leeuwen (2008) pour analyser la multimodalité de la communication visuelle et au cadre d'analyse de données présenté en anthropologie visuelle par Rose (2007) pour examiner les images, les sites de production vidéo, et les sites de visionnement des vidéos. Ainsi, nous comptons nous attarder sur les personnes, les événements et les lieux représentées dans les vidéos, en considérant aussi les discours, les thèmes, les genres de récit, les sons et tout autre aspect pertinent du champ visuel y compris la communication non-verbale des personnes visibles, leurs mouvements, la direction de leurs regards, etc., afin d'exploiter au maximum les données visuelles.

Pour montrer l'intérêt heuristique que peut présenter l'application d'une perspective critique à l'analyse des données visuelles, deux questions ont orienté notre examen préliminaire des extraits retenus ici : 1) quelles ressources matérielles et sociales ont été mobilisées dans la production des vidéos ; 2) comment la distribution du pouvoir étaitelle impliquée dans la sélection des informations présentées dans la vidéo et dans les rôles adoptés par les enfants pendant la production des vidéos? Dans la discussion qui suit, nous nous concentrons sur les vidéos produites dans le contexte mexicain pour indiquer comment une pédagogie axée sur les littératies multimodales laisse place à la reconnaissance du répertoire plurilingue des enfants et prend en compte leurs connaissances intimes de leur quartier et des pratiques culturelles de leur famille et communauté, des ressources peu exploitées à l'école. Nous proposons aussi que, pour comprendre la portée d'une telle innovation, l'analyse du produit vidéo ne suffit pas, mais qu'elle doit être accompagnée d'une interprétation d'autres données fournissant des informations contextuelles. Raison pour laquelle, en plus d'analyser le produit vidéo, nous nous penchons sur la triangulation des différentes sources de données, y compris les photos prises pendant la production, les notes de terrain et les textes écrits par les étudiants-maitres. Cette triangulation des données rend possible l'adoption d'un regard plus holistique et permet de développer une compréhension plus nuancée à la fois du processus de production et des dynamiques de pouvoir en place afin d'identifier quelles sont les possibilités et les limites de l'exploitation de la vidéo dans les activités de littératies multimodales. 
30 Les activités de production vidéo au Mexique ont été possibles grâce à une étroite collaboration avec le Dr. Mario López Gopar, chercheur en linguistique appliquée et professeur d'université qui s'intéresse au plurilinguisme et aux littératies multimodales et qui a facilité leur intégration dans des cours bilingues d'anglais et de zapotèque offerts dans une bibliothèque communautaire qu'il dirige. La bibliothèque était située dans une petite maison en béton, où les murs de la pièce principale étaient remplis de rayons de livres unilingues et bilingues en anglais, espagnol et zapotèque, avec une section dédiée à des livres plurilingues. Dix enfants de familles hispanophones du quartier ouvrier environnant la bibliothèque ont participé à ces activités après l'école. Trois étudiants mexicains inscrits en formation des maîtres à l'université locale qui faisaient un stage d'observation/enseignement à la bibliothèque sous la supervision du Dr. López Gopar ont collaboré avec les cinq stagiaires canadiens pour mettre en place ces activités.

31 Au début du projet, les enfants ont participé à un remue-méninges oral pour choisir les lieux qu'ils voulaient représenter dans leur vidéo. Après avoir identifié plusieurs sites à filmer, ils ont décidé par vote de retenir les quatre lieux suivants : la bibliothèque, l'école primaire du quartier à laquelle ils étaient inscrits, l'église catholique du quartier et le terrain de sport universitaire situé à quelques rues de la bibliothèque, accessible au grand public. Dans cette discussion, les enfants ont aussi négocié qui allait paraître sur le film. Ils se sont mis d'accord sur le fait que chaque enfant allait se présenter à tour de rôle à la bibliothèque et que certains élus allaient servir d'animateur pour présenter les lieux dans la vidéo. Les enfants ont ensuite collaboré en petits groupes pour produire les scénarios en espagnol et en anglais pour guider la présentation des lieux et les animateurs ont appris ces textes par cœur en vue de les réciter durant le tournage.

Cette collaboration a débouché sur la production d'une vidéo de 21 minutes et d'une version plus courte de celle-ci qui dure 7 minutes. Dans la version longue, un titre bilingue apparait au début de chaque scène et il est parfois accompagné d'un sous-titre. Par exemple, le nom de la bibliothèque figure au bas de l'écran au début de la première scène avec le sous-titre "Biblioteca Comunitaria - Community Library " présenté en dessous. La vidéo est organisée en huit scènes présentant les enfants dans divers lieux de la communauté. La première scène dure environ deux minutes et commence par un long plan séquence des enfants et des stagiaires assis en cercle sur un tapis dans le local principal de la bibliothèque (voir les clips présentés dans l'Annexe 1). Les enfants dessinent dans leur cahier pendant que les stagiaires les observent, parlent avec eux et regardent leurs dessins. Des plans plus rapprochés permettent de voir que les dessins illustrent des objets avec des mots anglais écrits en dessous. On entend des mots en espagnol et en anglais prononcés par les enfants et les stagiaires, entrecoupés par des rires d'enfants. Les plans suivants montrent les enfants et les stagiaires qui jouent à un jeu kinésique et silencieux debout sur le tapis. Ensuite, quelques séquences courtes montrent deux des stagiaires mexicaines qui tiennent à tour de rôle un dessin d'enfant et lisent ce qui est écrit dessus, comme par exemple, "I like chocolate cake » et des plans plus proches focalisés sur ces dessins. Dans la deuxième scène de la vidéo, d'une durée d'environ huit minutes, chaque enfant se présente à tour de rôle, assis face à la caméra. Certains parlent en anglais et d'autres en espagnol pour dire leur nom, où ils vivent, leur âge et pour nommer les membres de leur famille en présentant une photo d'elle. Quelques enfants s'appuient sur un texte écrit pour parler et les parties de la vidéo où ils parlent en espagnol sont sous-titrées en anglais. La troisième scène ouvre avec une vue de deux garçons du groupe debout devant une fresque murale très colorée situé le long d'une rue 
près de la bibliothèque qui annonce les parades prévues pour la Fête des Morts dans les prochains jours. Dans un clip qui dure onze secondes, les garçons improvisent une explication en espagnol de la façon dont ces jours sont célébrés dans leur communauté et leur famille. La quatrième scène d'environ deux minutes est consacrée à un tour guidé de l'extérieur de l'école du quartier mené en anglais par trois filles, suivi de clips où on voit l'ensemble des enfants jouer au basketball sur le terrain scolaire. La cinquième scène d'environ trois minutes présente des clips d'un tour guidé de l'intérieur de l'église mené en anglais d'abord et en espagnol ensuite par trois garçons du groupe, ensuite un clip montre un des garçons qui présente le prêtre assis dans son bureau, suivi d'un clip figurant le carillonneur qui tire sur une corde pour sonner la cloche de l'église. Dans les premiers clips de la sixième scène, qui dure environ quatre minutes, trois filles sont debout sous un grand arbre dans le terrain de jeu de l'université et elles présentent une vue des montagnes autour de la ville en expliquant en espagnol quels sports sont pratiqués sur le terrain. Dans le clip suivant, animé par de la musique mexicaine, tous les enfants participent à des jeux sportifs tels que des courses à pied. Ensuite la septième scène montre sur 40 secondes une vue de dos des enfants et des adultes qui marchent le long de la rue pour retourner à la bibliothèque et dans la huitième scène, d'une durée d'environ une minute, on voit les enfants peindre un panneau mural sur l'extérieur de la bibliothèque.

Dans une analyse préliminaire de la vidéo, on peut observer que dès la première scène où chaque élève s'est présenté en anglais ou en espagnol selon sa préférence et qu'il a partagé des informations orales et visuelles sur sa famille, les enfants avaient accès à plusieurs ressources pour s'exprimer. On note qu'ils pouvaient puiser dans les langues de leur répertoire pour communiquer et que cela a rempli deux fonctions pédagogiques. Premièrement, cela a servi à signaler la légitimité du plurilinguisme dans ce lieu d'apprentissage des langues et leur a permis de montrer aux spectateurs qu'ils avaient acquis un certain niveau de compétence dans la langue choisie. L'opportunité de se référer à un texte écrit au besoin et de présenter des photos de famille a offert aux jeunes un support sur lequel ils pouvaient s'appuyer en parlant, si nécessaire, et une façon de transmettre rapidement des informations qui les situent au sein d'une famille en donnant des indices visuels sur leur position dans ce groupe ainsi que sur les similitudes et les différences entre les membres de la famille, sans avoir à décrire tout cela à l'oral. Deuxièmement, la vidéo leur a fourni un moyen d'exprimer par des gestes et des sourires leur sentiment de fierté et d'attachement à leur famille. Ainsi, les enfants ont pu mobiliser des ressources linguistiques, matérielles, sociales et gestuelles pour communiquer leurs messages.

34 Par ailleurs, la vidéo a donné aux enfants une occasion d'étaler leurs habiletés de récitation et leurs capacités à partager des connaissances sur leur quartier. Ils ont pu communiquer leur compréhension de pratiques culturelles, telles que les sports pratiqués sur le terrain de l'école et de l'université, les préparatifs dans leurs familles et leur communauté pour la Fête des Morts, et l'importance accordée à la religion catholique dans leur milieu. Ci-dessous, l' extrait de la transcription audio de la vidéo indique que pour effectuer les tours guidés des lieux filmés, les enfants ont emprunté un genre de discours typiquement utilisé dans les programmes de voyage et les documentaires qui passent à la télévision. Une traduction en français de cette transcription figure dans l'Annexe 2.

Extrait 1 
Trois garçons sont debout à l'entrée de l'église.

Garçon 1: Welcome to church Catholic.

Garçons 1 et 2 : We are Catholic.

Garçons 1, 2 et 3 font quelques pas dans l'église, s'arrêtent pour regarder la caméra et dire : Este es la iglésia dondé todos los días, exepto lunes, se hace la misa.

Garçon 1 marche vers l'allée droite dans l'église en disant: Nosotros les enseñaremos algunos santos.

Garçon 1 montre une statue en disant: El fue un amigo de Dios. Fue un gran profeta.

Garçons 1, 2 et 3 montrent une autre statue en disant : Este es San Francisco de Azis.

Vue de dos des trois garçons qui avancent vers l'autel. On entend la voix d'un des garçons qui annonce: $\mathrm{Y}$ estos son unos de los santos y ahora les presentaremos el más importante de todos.

Garçons 1, 2 et 3 debout devant l'autel montrent une statue du Christ sur la croix située derrière l'autel: Ese es Jesús, fue crucificado para perdonar nuestros pecados.

(Transcription audio du segment vidéo filmé dans l'église, minutes 13 :29 à $14: 39)$ pour accueillir les spectateurs à l'église et s'identifier en tant que catholiques, puis en passant assez rapidement à l'espagnol pour adopter un discours religieux et offrir une explication plus détaillée de ce que représentent les statues situées dans cet endroit. Si on examine à partir d'une perspective critique quelles ressources ont été mobilisées par les enfants pour produire ce message, on peut considérer que la participation à la production de la vidéo est une activité prometteuse car elle rend perméable la frontière entre l'école et la communauté en permettant aux enfants d'inclure non seulement leurs ressources bilingues, mais aussi les connaissances acquises en contexte hors scolaire sur les pratiques religieuses et culturelles adoptées dans l'environnement local. Comme l'a révélé la recherche de Brass (2008), de tels projets vidéo stimulent l'intégration des connaissances locales, la recontextualisation du matériel culturel et l'imbrication de l'apprentissage officiel et non officiel. On peut aussi considérer que l'ancrage des informations orales dans le champ visuel repose sur une approche plus équitable car il ne privilégie pas un seul mode d'expression, mais il facilite plutôt le recours à plusieurs ressources sémiotiques parmi lesquelles les enfants ont pu faire une sélection selon leurs besoins et leurs habiletés pour attirer l'attention, transmettre un message stimulant et se positionner de façon puissante comme des individus dotés de connaissances.

Par contre, comme l'indiquent les notes de terrain suivantes, le potentiel inclusif d'une telle activité axée sur les littératies multimodales peut être limité quand les pratiques représentées dans la vidéo correspondent surtout à celles qui sont privilégiées par la majorité de la communauté et ne reflètent pas nécessairement celles adoptées par les groupes minorisés. Dans cet extrait, deux stagiaires mexicaines expliquent qu'elles ne sont pas catholiques.

Extrait 2

J'ai rencontré dans la rue deux stagiaires mexicaines qui attendaient l'ouverture de la bibliothèque. En mélangeant l'anglais et l'espagnol, nous avons parlé des préparatifs pour la Fête des Morts. Elles m'ont raconté qu'elles ne pratiquent pas ces rituels puisqu'elles sont protestantes et qu'elles vont à une église évangélique située près de la bibliothèque. Je leur ai demandé combien de membres de leur famille ne sont pas catholiques et, 
si j'ai bien compris ce qu'elles m'ont dit, toute la famille d'une des jeunes filles est catholique sauf elle, sa mère, ses frères et sœurs. C'est la même chose pour l'autre, sauf que sa grand-mère est protestante aussi.

(Notes de terrain, 28 octobre 2010) n'était pas représenté dans la vidéo et elles soulignent comment les dynamiques entre groupes majoritaires et minorisées peuvent être reproduites dans ce qui est visible et invisible dans le produit. Rappelons que les enfants ont décidé par vote quels lieux ils allaient filmer, alors cette procédure bien démocratique visant à donner voix à chaque élève dans la prise de décision lors des activités de production, aurait aussi pu renforcer le pouvoir de la majorité. De plus, il est possible que la sélection de certaines personnes pour présenter les informations pendant le tournage aurait peut-être servi à mettre en valeur et rendre plus visible ceux qui se sentaient à l'aise et s'exprimaient bien en public et à marginaliser davantage ceux qui n'avaient pas ces mêmes dons. À l'inverse, comme le suggère l'observation suivante d'une des stagiaires, le processus de production de vidéo, qui s'est étalé sur plusieurs semaines, a permis à chaque enfant de collaborer et de contribuer d'une façon ou d'une autre à diverses étapes de la construction du produit final. L'Annexe 3 présente une traduction en français de cet extrait.

Extrait 3

It was great to see the students so centred on the project. On our major filming day each student came prepared with the props and necessary equipment needed to film each scene. It gave the group a common goal to work towards and had all students interacting with each other, the teachers, and interacting with the camera. I feel that the video making procedure does involve all the students but at different levels. Many students were timid when speaking in front of the camera, giving way to those who were more comfortable.... Despite this... each student had a say in what was scripted and filmed. The students were collaborating on the storyboarding, which allowed for all... to be involved.

(Texte rétrospectif, étudiant-maître C., p. 4, le 5 décembre 2010)

\section{Conclusions}

Nous avons vu que les développements théoriques ces dernières années ont contribué à élargir la compréhension de la littératie comme pratique complexe englobant divers modes d'expression. Grâce aux résultats des études sur les fonctions de la littératie menées dans différentes sociétés, celle-ci est vue aujourd'hui comme un processus social de construction de sens qui varie en fonction du contexte. En portant l'attention sur les pratiques situées des acteurs sociaux, les recherches issues du courant Nouvelles études de la littératie et l'introduction de notions comme les littératies multiples ont signalé qu'en plus d'être variés, les discours sur la littératie et les actions qui y sont associées sont idéologiques et servent soit à reproduire les inégalités, soit à faire émerger des pratiques plus diversifiées et mieux adaptées aux besoins de populations de plus en plus hétérogènes. Les notions de littératies plurilingues et de répertoires plurilittératiés introduits plus récemment indiquent que plusieurs langues et formes scripturales caractérisent la communication et peuvent être mobilisées en fonction des exigences du contexte. Le terme littératies multimodales souligne que les messages sont communiqués et interprétés par le biais de plusieurs sens et à l'aide de différents modes sémiotiques où 
l'oral et l'écrit sont intégrés avec d'autres modes visuels et auditifs et que ces formes de communication se multiplient et changent continuellement avec le développement des outils informatiques et de télécommunication.

Bien que notre analyse de données provenant de notre recherche ne peut être que très préliminaire à ce stade-ci du projet, nous avons tenté d'illustrer, à l'aide de quelques extraits de données, qu'une perspective critique appliquée à l'étude des littératies multimodales fournit un outil heuristique intéressant et identifie des pistes d'analyses prometteuses qui méritent d'être poursuivies dans des analyses ultérieures. Cette perspective permet d'expliquer comment les conditions mises en place dans une situation pédagogique où l'expression visuelle est permise encouragent les enfants à explorer plusieurs moyens de se représenter et de communiquer en ouvrant des portes sur l'intégration des connaissances hors scolaires et des pratiques adoptées dans la communauté. Notre discussion de ce projet de production vidéo a montré aussi que l'inclusion des formes d'expression visuelles dans l'enseignement et l'adoption de démarches démocratiques dans les prises de décision en groupe ne garantissent pas nécessairement que les processus pédagogiques seront plus équitables ou que les produits vidéos présenteront des images qui reflèteront la réalité de tous. Durant l'implantation de projets semblables dans l'avenir, il serait important d'allouer assez de temps dans la phase initiale de production vidéo à l'émergence des idées divergentes provenant de tous les participants, y compris ceux qui sont plus marginalisés dans les interactions, et de porter attention à la façon dont l'équipe de production négocie ce qui est retenu dans la création du produit final afin qu'il reflète les contributions de la collectivité dans toutes ses différences. Maintenir l'équité dans la distribution du pouvoir entre les participants impliqués dans la production vidéo et assurer la représentativité des contenus retenus dans les produits visuels issus de ce processus semblent demeurer des défis de taille auxquels il faut veiller dans ces activités afin d'identifier quelles sont les limites et les possibilités de telles innovations. Une perspective critique peut servir à cerner où elles se situent car elle attire l'attention sur les tensions et les contradictions qui émergent parfois entre les objectifs d'un projet pédagogique comme la production vidéo et les dynamiques sociales en place sur le terrain, ce qui permet en même temps d'éclairer les pistes les plus prometteuses à suivre dans l'avenir pour contourner les obstacles et agir autrement.

\section{BIBLIOGRAPHIE}

Apple, M. (1979). Ideology and Curriculum. London : Routledge.

Ajayi, L. (2009). « English as a second language learners' exploration of multimodal texts in a junior High school ». Journal of Adolescent and Adult Literacy, vol. 52, n 7, pp. 585-595.

Armand, F. (2000). « Le rôle des capacités et de la compétence langagière orale dans l'apprentissage de la lecture en français langue maternelle et seconde ». La Revue canadienne des langues vivantes, vol. $56, \mathrm{n}^{\circ} 3$, pp. 469-495. 
Auerbach, E. (1989). « Toward a social-contextual approach to family literacy ». Harvard Educational Review, vol. 59, n², pp. 165-182.

Bourdieu, P. \& Passeron, J.C. (1970). La reproduction : éléments pour une théorie du système d'enseignement. Paris : Éditions de Minuit.

Billiez, J. \& Tremaille, C. (2001). « Plurilinguisme, variations, insertion scolaire et sociale », Langage et Société, vol. 98, n 4, pp. 105-127.

Billiez, J., Candelier, M., Costa-Galligani, Lambert, P., Sabatier C. \& Trimaille, C. (2003). « Contacts de langues à l'école : disjonctions et tentative de raccordements ». In J. Billiez (dir.). Contacts de langues : modèles typologies, interventions. Paris : l'Harmattan.

Brandt, D. \& Clinton, K. (2002) « Limits of the local : Expanding perspectives on literacy as a social practice ». Journal of Literacy Research, vol. 34, $n^{\circ} 3$, pp. 337-356.

Brass, J.J. (2008). « Local knowledge and digital movie composing in an after-school literacy program ». Journal of Adolescent and Adult Literacy, vol. 51, $\mathrm{n}^{\circ}$ 6, pp. 484-478.

Brice, S. \& Street, B. (2008). Ethnography : Approaches to Language and Literacy Research. New York : Teacher's College Press.

Bruce, D. L. (2009). « Reading and writing video : Media literacy and adolescents ». In Christenbury, L. Bomer, R. \& Smagorinsky, P. (dir.). Handbook of Adolescent Literacy Research. New York : The Guilford Press.

Carrington, V. \& Marsh, J. (2005). « Digital childhoods and youth : New texts, new literacies. Discourse »: Studies in the Cultural Politics of Education, vol. 26, n 3, pp. 279-285.

Chiss, J.-L. (2003). « La littératie : quelques enjeux d'une réception dans le contexte éducatif et culturel français ». In C. Barré-De Miniac et al. (dir.). La littéracie. Conceptions théoriques et pratiques d'enseignement de la lecture-écriture. Paris : L'Harmattan.

Cope, B. \& Kalantzis, M. (2000). Multiliteracies : Literacy Learning and the Design of Social Futures. New York : Routledge.

Coste, D., Moore, D. \& Zarate, G. (1997). « Compétence plurilingue et pluriculturelle. Le Français dans le Monde/ Recherches et Applications ». Numéro spécial : Apprentissage et usage des langues dans le cadre européen, pp. 8-67.

Cummins, J. (2006). «Identity texts : The imaginative construction of self through multiliteracies pedagogy ». In Garcia, O. Skutnabb-Kangas, T. \& Torres- Guzman, M. (dir.). Imagining multilingual schools : Language in education andglocalization. Clevedon, UK : Multilingual Matters.

Cunningham, J., Many, J., Carver, R., Gunderson, L. \& Mosenthal, P. (2000). « Snippets : How will literacy be defined?» Reading Research Quarterly, vol. 35, n 1, pp. 64-71.

Dagenais, D. (2008). « Pratiques langagières des enfants de familles immigrantes à la maison et à l'école ». Revue des sciences de l'éducation, vol. 34, n², pp. 351-376.

Dagenais, D. \& Day, E. (1999). « Home language practices of trilingual children in French Immersion. » La revue canadienne des langues vivantes, vol. 56, $\mathrm{n}^{\circ}$ 1, pp. 99-123.

Dagenais, D. \& Day, E. (1998). « Classroom language experiences of trilingual children in French immersion. » The Canadian Modern Language Review, vol. 54, n 3, pp. 376-393.

Dagenais, D. \& Moore, D. (2008). « Représentations des littératies plurilingues, de l'immersion en français et des dynamiques identitaires chez des parents en chinois. » Revue canadienne des langues vivantes, vol. $65, \mathrm{n}^{\circ} 1$, pp. 11-32. 
Debruin-Parecki, A. \& Klein, H. A. (2003). « Stvaranje Prijatelja/Making Friends : Multimodal literacy activities as bridges to intercultural friendship and understanding in a cross-cultural collaborative literacy project ». Journal of Adolescent \& Adult Literacy, vol. 46, n 6, pp. 506-513.

Ferdman, B. (1990). « Literacy and cultural identity ». Harvard Educational Review, vol. 60, n² 2, pp. 181-204.

Flewitt, R. (2008). « Multimodal literacies ». In Marsh, J. \& Hallet, E. (dir.). Desirable Literacies : Approaches to Language and Literacy in the Early Years. London : Sage.

Flood, J, Heath, S.B. \& Lapp, D. (2008). Handbook of Research on Teaching Literacy through the Communicative and Visual Arts II : A Project of the International Reading Association. New York: Lawrence Earlbaum.

Fraenkel, B. \& Mbodj, A. (2010). « Introduction. Les New Literacy studies, jalons historiques et perspectives actuelles. » Langage et société, vol. 33, n 3, pp. 7-24.

Freire, P. (1970). Pedagogy of the Oppressed. New York : Continuum.

Giroux, H.A. (1983). « Theories of reproduction and resistance in the new sociology of education : A critical analysis ». Harvard Educational Review, vol. 53, n 3, pp. 257-293.

Gregory, E. \& Williams, A. (2000). « Work or play? Unofficial literacies in two East London communities ». In Martin-Jones M. \& Jones K. (dir.). Multilingual Literacies. Philadelphia : John Benjamins.

Grosjean, F. (1993). « Le bilinguisme et le biculturalisme : essai de définition ». Tranel, vol. 19, pp. 13-42.

Halliday, M.A.K. (1994). An Introduction to Functional Grammar. London : Edward Arnold.

Halliday, M.A.K. (1978). Language as Social Semiotic. London : Edward Arnold.

Hass Dyson, A. (2001). « Coach Bombay's kids learn to write : Children's appropriaton of media material for school literacy ». In Cushman, E. Kintgen, E. Kroll B. \& Rose M. (dir.). Literacy : A critical sourcebook. Boston : Bedford/ St. Martin's.

Heath, S.B. (1983). Ways with Words : Language, Life and Work in Communities and Classrooms. New York : Cambridge University Press.

Heath, S.B. \& Street, B. (2008). On Ethnography: Approaches to Language and Literacy Research. New York : Teachers College Press.

Heath, S. B. \& McLaughlin, M. W. (dir.) (1993). Identity and Inner-city Youth. New York : Teachers College Press.

Hull, G. \& Schultz, K. (2002). School's out! Bridging out-of-school Literacies with Classroom Practice. New York : Teachers College Press.

Hull, G. \& Nelson, M. E. (2005).« Locating the semiotic power of multimodality ». Written Communication, vol. 22, $\mathrm{n}^{\circ}$ 2, pp. 224-261.

Ibrahim, A. (1999). Becoming Black : « Rap and Hip-Hop, Race, Gender, Identity and the Politics of ESL Learning ». TESOL Quarterly, vol. 33, n 3, pp. 349-371.

Jewitt, C. \& Kress, G. (dir.) (2003). Multimodal Literacy. New York : Peter Lang.

Kendrick, M., Rogers, T., Toohey, K., Marshall, E., Mutonyi, H., Hauge, C., Siegel, M. \& Rowsell, J. (2010). «Experiments in visual analysis : (Re)positions of children and youth in relation to larger sociocultural issues ». National Reading Conference Yearbook, vol. 59, pp. 395-408. 
Kendrick, M., \& McKay, R. (2002). « Uncovering literacy narratives through children's drawings ». Canadian Journal of Education, vol. 27, $\mathrm{n}^{\circ} 1$, pp. 45-60.

Kress, G. (2000). « Multimodality ». In Cope B. \& Kalantzis M. (dir.). Multiliteracies : Literacy Learning and the Design of Social Futures. London : Routledge.

Kress, G. (2003). Literacy in the new Media Age. London : Routledge.

Kress, G. \& van Leeuwen, T. (1996). Reading Image s: The Grammar of Visual Design. London : Routledge.

López Gopar, M. (2007). Beyond the alienating alphabetic literacy : Multiliteracies in indigenous education in Mexico. Diaspora, Indigenous, and Minority Education, vol. 1, n 3, pp. 159-174.

Lotherington, H., Sotoudeh, S., Holland, M. \& Zentena, M. (2008). Teaching emergent multiliteracies at Joyce Public School : Three narratives of multilingual story-telling in the primary grades. Canadian Modern Language Review, vol. 65, n 1, pp. 125-145.

Marsh, J. (2006). « Global/local/public, private : Young children's engagement in digital literacy practices in the home ». In Pahl K. \& Rowsell J. (dir.). Travel Notes from the New Literacy Studies : Instances of Practice. Clevedon, UK : Multilingual Matters.

Marshall, E. \& Toohey, K. (2010). Representing family : Community funds of knowledge, bilingualism and multimodality. Harvard Educational Review, vol. 80, n² 2, pp. 221-241.

Martin-Jones, M. \& Jones, K. (2000). Multilingual Literacies. Philadelphia, PA : John Benjamins.

Moore, D. (2006). Plurilinguismes et école. Paris : Didier.

New London Group. (1996). « A pedagogy of multiliteracies : Designing social futures ». Harvard Educational Review, vol. 66, n 1, pp. 60-92. Réimprimé dans Cope B. \& Kalantzis M. (dir.) (2000). Multiliteracies : Literacy Learning and the Design of Social Futures. London : Routledge.

Norton, B. \& Toohey, K. (dir.) (2004). Critical Pedagogies and Language Learning. Cambridge : Cambridge University Press.

Painchaud, G., d'Anglejan, A., Armand, F. \& Jesak, M. (1993). Diversité culturelle et littératie. Repères : Essais en éducation, vol. 15, pp. 77-94.

Pennycook, A. (2001) Critical Applied Linguistics : A Critical Introduction. Mahwah, NJ : Lawrence Erlbaum Associates.

Prinsloo, M. (2005). The new literacies as placed resources. Perspectives in Education, vol. 23, $\mathrm{n}^{\circ} 4$, pp. 87-98.

Reuter, Y. (2003). La littératie. Perspectives pour la didactique. LIDIL, 27, 11-23.

Rogers, T., Winters, K., LaMonde, A.M. \& Perry, M. (2010). From image to ideology : Analyzing shifting identity positions of marginalized youth across the cultural sites of video production. Pedagogies: An International Journal, vol. 5, n 4, pp. 298-312.

Rose, G. (2007). Visual Methodologies : An Introduction to the Interpretation of Visual materials. $2^{\text {nd }}$ Edition. London : Sage.

Stein, P. (2004). « Representation, rights, and resources : Multimodal pedagogies in the language and literacy classroom ». In Norton B. \& Toohey K. (dir.). Critical Pedagogies and Language Learning. Cambridge, UK : Cambridge University Press.

Smythe, S. \& Toohey, K. (2009a). Investigating sociohistorical contexts and practices through a community scan : A Canadian Punjabi Sikh example. Language and Education, vol. 21, $\mathrm{n}^{\circ} 1$, pp. 37-57. 
Smythe, S. \& Toohey, K. (2009b). « Bringing home and community to school : Institutional constraints and pedagogic possibilities ». In Miller, J. Kostogriz A. \& Gearon M. (dir.). Culturally and Linguistically Diverse Classrooms : New Dilemmas for Teachers. Clevedon, UK : Multilingual Matters.

Street B. (2000), « Literacy events and literacy practices. Theory and practice in the New Literacy Studies ». In Martin-Jones M. \& Jones K. (dir.). Multilingual Literacies. Philadelphia, PA : John Benjamins.

Street, B. (2003). « What's "new" in New Literacy Studies? Critical approaches to literacy in theory and practice ». Current Issues in Comparative Education, vol. 5, n² 2, pp. 77-91.

Toohey, K., Dagenais, D. \& Schulze, E. (soumis). Making video with second language learners. Van Enk, A., Dagenais, D. \& Toohey, K. (2005). « Contextualizing literacy and learning : Some emerging considerations for a socio-cultural perspective on school-based research. » Language and Education, vol. 19, n 6, pp. 496-512.

Van Leeuwen, T. (2008). Discourse and Practice: New Tools for Critical Discourse Analysis. Oxford: Oxford University Press.

Wells, G. (1986). The Meaning Makers : Children Learning Language and Using Language to Learn. Portsmouth, NH : Heinemann.

\section{ANNEXES}

\section{Annexe 1}

Vidéoclips filmés à la bibliothèque et dans la rue

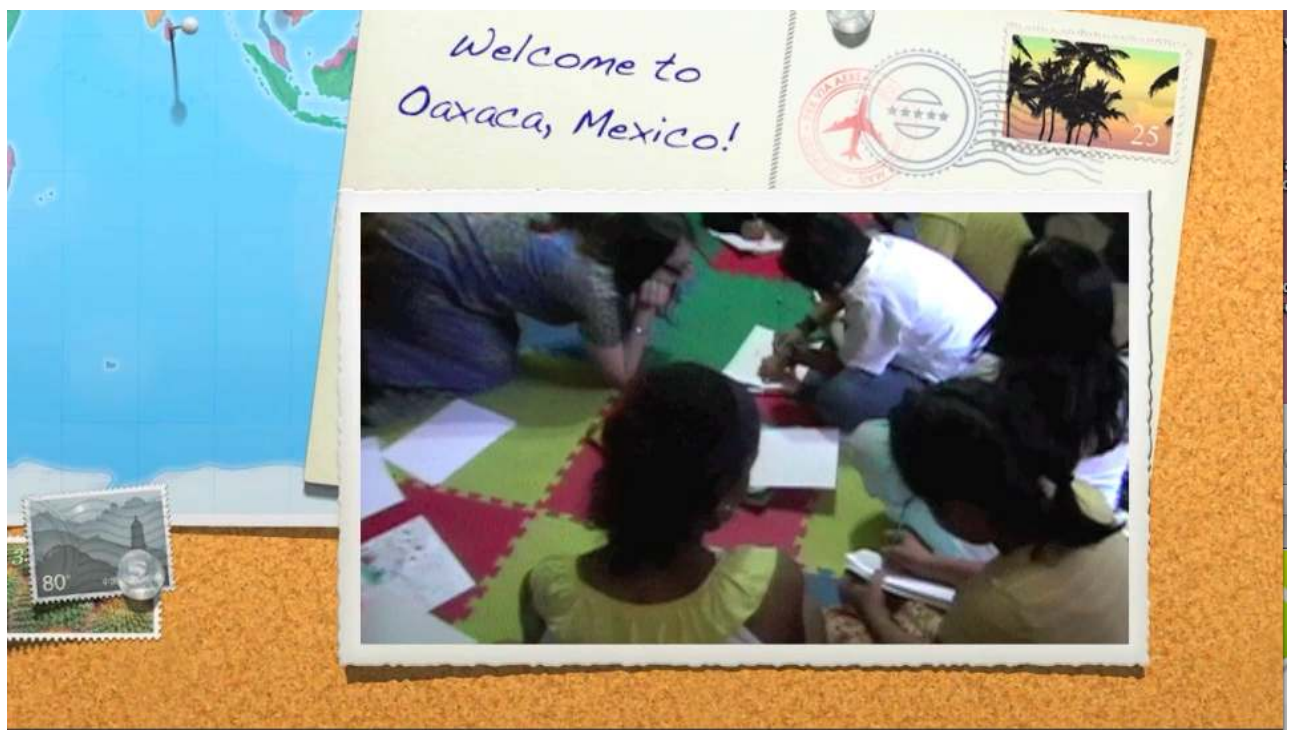




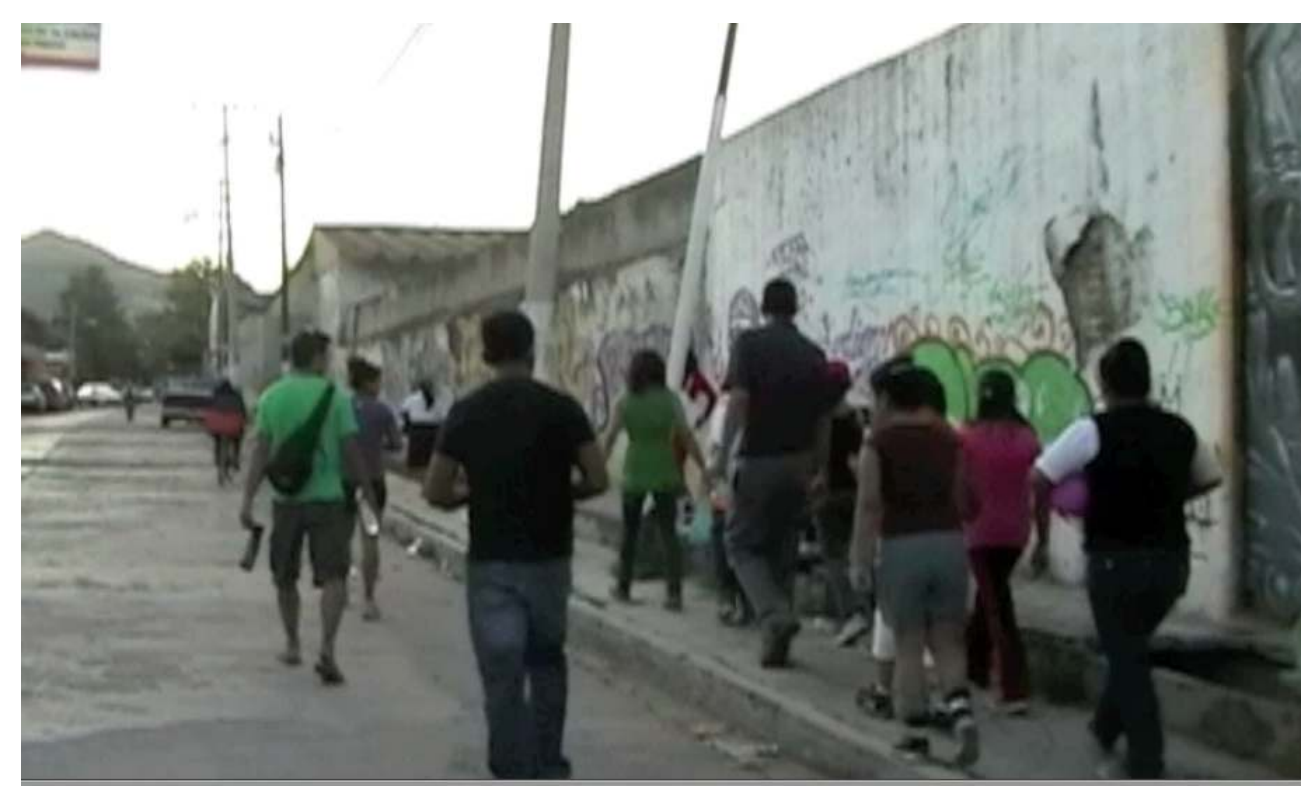

\section{Annexe 2}

Traduction de l'extrait 1

Trois garçons sont debout à l'entrée de l'église.

Garçon 1 (en anglais) : Bienvenue à l'église catholique.

Garçons 1 et 2 (en anglais) : Nous sommes catholiques.

Garçons 1, 2 et 3 prennent quelques pas dans l'église, s'arrêtent pour regarder la caméra et dire en espagnol : Voici l'église où il $\mathrm{y}$ a des messes tous les jours sauf le lundi.

Garçon 1 marche vers l'allée droite dans l'église en disant en espagnol : Nous allons vous montrer des objets saints.

Garçon 1 montre une statue en disant en espagnol : C'était un ami de Dieu. Il était un grand prophète.

Garçons 1, 2 et 3 montrent une autre statue en disant en espagnol : C'est François d'Assise.

Plan de dos des trois garçons qui avancent vers l'autel. On entend la voix d'un des garçons qui annonce en espagnol : Voici quelques-uns des autres saints et maintenant nous allons vous montrer le plus important de tous.

Garçons 1, 2 et 3 devant l'autel montrent une statue du Christ sur la croix située derrière l'autel (en espagnol) : Voici Jésus qui a été crucifié pour pardonner nos fautes

(Transcription audio du segment vidéo filmé dans l'église, minutes 13 : 29-14 : 39).

\section{Annexe 3}

Traduction de l'extrait 3

C'était formidable de voir les enfants si centrés sur le projet. Lors de la principale journée de tournage, chaque étudiant est arrivé préparé avec les profs et l'équipement nécessaire pour filmer chaque scène. Cela a donné au groupe un objectif commun sur lequel travailler et ça a mené tous les élèves à interagir entre eux, avec les enseignants, et avec la caméra. Je sens que la production vidéo implique tous les élèves mais à différents niveaux. Plusieurs élèves étaient timides en parlant devant la caméra et ils ont cédé la 
place à ceux qui étaient plus à l'aise... En dépit de cela... chaque élève avait son mot à dire dans ce qui paraissait dans le scénario et était filmé. Les étudiants collaboraient sur le scénarimage, ce qui leur a permis à tous... d'être impliqués (Texte rétrospectif, étudiantmaître C., p. 4, le 5 décembre 2010).

\section{RÉSUMÉS}

Cet article examine l'évolution du concept de littératie ces dernières années et plus particulièrement des notions de littératies multiples, littératies plurilingues, répertoire plurilittératié, multimodalité et littératies multimodales qui circulent dans les écrits en anglais et dans quelques publications récentes en français. La discussion signale comment les recherches sur l'apprentissage des pratiques de littératie basées sur une perspective critique ont contribué à identifier des pistes pédagogiques plus équitables offrant aux enfants de diverses origines linguistiques et culturelles une plus grande variété de moyens de s'exprimer, de se représenter et de développer leurs pratiques de communication dans une société de plus en plus hétérogène et technologisée. Dans ce texte, la perspective critique est appliquée à l'étude de quelques extraits de données provenant d'un projet de production et d'échange de vidéos mené auprès d'enfants bilingues et plurilingues vivant dans différents pays afin d'illustrer comment une analyse critique peut servir d'outil heuristique pour identifier quelles conditions matérielles et sociales facilitent ou nuisent à l'apprentissage dans une innovation pédagogique axée sur les littératies multimodales.

This article examines how literacy has developed as a construct in recent years and in particular how terms such as multiliteracies, multilingual literacies, multiliterate repertoire, multimodality and multimodal literacies have evolved in English language scholarship and in some recent publications in French. The discussion highlights how research on literacy practices based on a critical perspective have contributed to identifying more equitable pedagogical approaches for learners of diverse linguistic and cultural origins. These pedagogies enable learners to represent themselves using a greater variety of modes of expression and develop their communicative practices in a society that is increasingly heterogeneous and technological. In this text, a critical perspective is applied to the analysis of a few data excerpts from a project focused on the production and exchange of videos between bilingual and multilingual children living in different countries. This illustrates how a critical perspective can serve as a heuristic device to identify which material and social conditions might facilitate or impede learning concerning pedagogical innovation focused on multimodal literacies.

\section{INDEX}

Mots-clés : Littératies multiples, littératies plurilingues, répertoire plurilittératié, multimodalité, littératies multimodales, perspective critique

Keywords : Multiliteracies, multilingual literacies, multiliterate repertoire, multimodality, multimodal literacies, critical perspective 


\section{AUTEUR}

\section{DIANE DAGENAIS}

Diane Dagenais est professeure titulaire à la Faculté d'Éducation de l'Université Simon Fraser où elle enseigne des cours en français et en anglais aux études supérieures, au premier cycle et en formation initiale. Ses recherches, subventionnées par plusieurs sources, y compris le Conseil de recherche en sciences humaines du Canada, se situent en linguistique appliquée et portent sur les liens entre la diversité linguistique et l'éducation. Elle s'intéresse plus particulièrement à l'enseignement des langues, au bilinguisme, au plurilinguisme et aux pratiques de littératie scolaires et hors scolaires.

Courriel dagenais@sfu.ca

Adresse : Simon Fraser University, Faculty of Education, 8888 University Drive, Burnaby BC V5A 1S6, Canada. 